\title{
Whole-exome characterization of pancreatic neuroendocrine tumor cell lines BON-1 and QGP-1
}

\author{
Timon Vandamme ${ }^{1,2,3}$, Marc Peeters', Fadime Dogan ${ }^{2}$, Patrick Pauwels ${ }^{4}$, \\ Elvire Van Assche ${ }^{3}$, Matthias Beyens ${ }^{1,3}$, Geert Mortier ${ }^{3}$, Geert Vandeweyer ${ }^{3}$, \\ Wouter de Herder ${ }^{2}$, Guy Van Camp ${ }^{3}$, Leo J Hofland ${ }^{2}$ and Ken Op de Beeck ${ }^{1,3}$ \\ ${ }^{1}$ Department of Oncology, University of Antwerp, Universiteitsplein 1, 2610 Wilrijk, Antwerp, Belgium \\ ${ }^{2}$ Section of Endocrinology, Department of Internal Medicine, Erasmus Medical Center, Dr. Molenwaterplein 50, \\ 3015GE Rotterdam, The Netherlands \\ ${ }^{3}$ Center of Medical Genetics, and ${ }^{4}$ Department of Pathology, University of Antwerp and Antwerp University \\ Hospital, Universiteitsplein 1, 2610 Wilrijk, Antwerp, Belgium
}

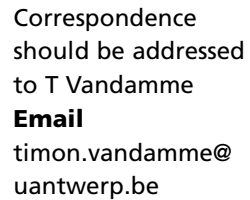

\begin{abstract}
The human BON-1 and QGP-1 cell lines are two frequently used models in pancreatic neuroendocrine tumor (PNET) research. Data on the whole-exome genetic constitution of these cell lines is largely lacking. This study presents, to our knowledge, the first wholeexome profile of the BON-1 and QGP-1 cell lines. Cell line identity was confirmed by short tandem repeat profiling. Using GTG-banding and a CytoSNP-12v2 Beadchip array, cell line ploidy and chromosomal alterations were determined in BON-1 and QGP-1. The exomes of both cell lines were sequenced on Ilumina's HiSeq next-generation sequencing (NGS) platform. Single-nucleotide variants (SNVs) and insertions and deletions (indels) were detected using the Genome Analysis ToolKit. SNVs were validated by Sanger sequencing. Ploidy of BON-1 and QGP-1 was 3 and 4 respectively, with long stretches of loss of heterozygosity across multiple chromosomes, which is associated with aggressive tumor behavior. In BON-1, 57 frameshift indels and 1725 possible protein-altering SNVs were identified in the NGS data. In the QGP-1 cell line, 56 frameshift indels and 1095 SNVs were identified. ATRX, a PNET-associated gene, was mutated in both cell lines, while mutation of TSC2 was detected in BON-1. A mutation in NRAS was detected in BON-1, while KRAS was mutated in QGP-1, implicating aberrations in the RAS pathway in both cell lines. Homozygous mutations in TP53 with possible loss of function were identified in both cell lines. Various MUC genes, implicated in cell signaling, lubrication and chemical barriers, which are frequently expressed in PNET tissue samples, showed homozygous proteinaltering SNVs in the BON-1 and QGP-1 cell lines.
\end{abstract}

Journal of Molecular Endocrinology (2015) 54, 137-147

\section{Introduction}

Pancreatic neuroendocrine tumors (PNETs) are malignant tumors probably arising from islet cells of the pancreas (Schimmack et al. 2011). PNETs can secrete a variety of hormones including somatostatin, insulin, glucagon, serotonin, and pancreatic polypeptide. The majority of PNETs, however, are nonsecreting (Yao et al. 2008). In the USA, the age-adjusted annual incidence of PNET is $0.32 / 100000$ and the standardized prevalence is http://jme.endocrinology-journals.org DOI: 10.1530/JME-14-0304
() 2015 Society for Endocrinology Printed in Great Britain
Published by Bioscientifica Ltd 
5/100 000, according to the Surveillance Epidemiology and End Results (SEER) data (Yao et al. 2008). In PNET, surgical resection alone is often curative in early-stage disease, but $40 \%$ to $50 \%$ of PNET patients have advanced disease at the time of initial diagnosis or present with synchronous tumors (Yao et al. 2008). Patients with advanced disease may suffer from complications of uncontrolled hormone secretion and usually succumb to these complications or tumor progression (Kulke et al. 2011).

PNETs are associated with a number of inherited syndromes that are characterized by mutations in wellstudied proto-oncogenes or tumor-suppressor genes. These include multiple endocrine neoplasia types 1 and 2 (MEN1 and MEN2), von Hippel-Lindau disease (VHL), and tuberous sclerosis (TSC). However, the majority of PNETs occurs as sporadic tumors (Leotlela et al. 2003).

To allow in vitro study of PNET, the BON-1, QGP-1, and CM human cell lines have been developed. Kaku et al. (1980) established the QGP-1 cell line from primary PNET tumor tissue obtained from a 61-year-old male. The BON-1 cell line was established by Townsend and colleagues. in 1986 from a peripancreatic lymph node metastasis of a 28-year-old male with PNET (Townsend et al. 1993). In 1987, the CM cell line was established from the peritoneal ascites of a patient with pancreatic insuloma (Baroni et al. 1999). The BON-1 cell line secretes neurotensin, pancreastatin, chromogranin A, serotonin (5-HT), 5-hydroxytryptophan (5-HTP), and 5-hydroxyindoleactic acid (5-HIAA) (Parekh et al. 1994). The QGP-1 cell line is a 5HT-, somatatostatin- and carcinoembryonic antigen (CEA)-secreting cell line (Iguchi et al. 1990, Doihara et al. 2009). Although BON-1 and CM have been karyotyped (Jonnakuty \& Gragnoli 2007, Lopez et al. 2010), highresolution whole-exome genetic information on PNET cell lines is currently lacking. In this study, we present genetic profiles of two frequently used PNET cell line models, BON-1 and QGP-1, obtained using genome-wide singlenucleotide polymorphisms (SNP) arrays and whole-exome sequencing. These profiles could lead to better insights into PNET tumorigenesis and identification of interesting new targets for therapy.

\section{Materials and methods}

\section{Cell culture and DNA isolation}

The QGP-1 cell line was purchased from the Japanese Collection of Research Bioresources Cell Bank (JRCB, Osaka, Japan). The BON-1 cell line was a kind gift from
Dr Townsend (University of Texas Medical Branch, Galveston, TX, USA). Both cell lines were confirmed as Mycoplasmafree using MycoAlert (Lonza, Verviers, Belgium). The BON-1 cell line was cultured in 1:1 mixture of DMEM and Ham's F-12K (Kaighn's) medium, supplemented with $10 \%(\mathrm{v} / \mathrm{v})$ FCS, penicillin $\left(1 \times 10^{5}\right.$ units/l), fungizone $(0.5 \mathrm{mg} / \mathrm{l})$, and L-glutamine ( $2 \mathrm{mmol} / \mathrm{l})$. The QGP-1 cell line was cultured in RPMI-1640 medium, supplemented with 10\% (v/v) FCS and penicillin-streptomycin $\left(1 \times 10^{5}\right.$ units/l). All cell lines were cultured in a humidified incubator at $5 \% \mathrm{CO}_{2}$ and $37^{\circ} \mathrm{C}$. The media and supplements were obtained from Life Technologies (Invitrogen). DNA from BON-1 (passage number 9) and from QGP-1 (passage number 14) was isolated using a QIAamp DNA Mini Kit (Qiagen), following the manufacturer's instructions. The concentration of the isolated DNA was quantified using a Qubit 2.0 fluorometer with a dsDNA Broad Range Assay (Thermo Scientific, Waltham, MA, USA). A high-molecular-weight band was visible on DNA gel electrophoresis, excluding DNA degradation. Short tandem repeat profiling using a Powerplex Kit (Promega) of BON-1 and QGP-1 gave results consistent with those obtained by the original group (Silva et al. 2011) and the JRCB database, respectively, thus confirming the cell lines' identity.

\section{SNP array}

BON-1 and QGP-1 DNA was hybridized on a CytoSNP12v2 Beadchip array (Illumina, San Diego, CA, USA), after whole-genome amplification with an Infinium HD assay (Illumina) using $200 \mathrm{ng}$ input DNA, according to the manufacturer's protocol. The array was scanned using an Illumina iScan (Illumina). The resulting scan data were imported, mapped, and clustered using Illumina GenomeStudio Software v2011.1 equipped with the genotyping module (version 1.9.4) (Illumina), before being exported for further analysis using allele-specific copy number analysis of tumors (ASCAT) (Van Loo et al. 2010) with default parameters. ASCAT takes both aneuploidy of the tumor cells and nonaberrant cell infiltration into account when analyzing SNP array data. This allows identification of copy number variants (CNVs), copy-number neutral events, and loss of heterozygosity (LOH) in nondiploid tumor samples, which cannot be picked up by noncorrected SNP array analysis algorithms.

\section{Preparation of metaphase spreads and GTG-banding}

Growth of BON-1 and QGP-1 cells in their respective media was synchronized by adding $100 \mathrm{nM}$ 5-fluor-

Published by Bioscientifica Ltd 
2-deoxyuridine and $4 \mu \mathrm{M}$ uridine overnight followed by $10 \mu \mathrm{M}$ thymidine after $15 \mathrm{~h}$. To prepare metaphase spreads, the synchronized cultures were arrested in mitosis by adding demecolcine $(1 \mu \mathrm{g} / \mathrm{ml})$ for $10 \mathrm{~min}$ at $37^{\circ} \mathrm{C}$. By incubating the cells in $75 \mu \mathrm{M} \mathrm{KCl}$ at $37^{\circ} \mathrm{C}$ for $20 \mathrm{~min}$, a hypotonic shock was applied after which the cells were fixed in methanol/acetic acid $(3: 1, \mathrm{v} / \mathrm{v})$ and washed twice. Metaphase spreads on uncoated glass slides were air-dried and thermally aged for $1 \mathrm{~h}$ at a temperature of $80-100{ }^{\circ} \mathrm{C}$. Giemsa-banding (GTG-banding) was executed using trypsin and eosin-polychromic-methylene blue solution (Leishman solution) according to the standard protocol (Seabright 1971). Chromosomal abnormalities identified by GTG-banding in 20 metaphases were described according to the guidelines of the International System for Human Cytogenetic Nomenclature (Shaffer et al. 2009).

\section{Whole-exome sequencing}

Two micrograms of isolated BON-1 and QGP-1 DNA were fragmented using a focused-ultrasonicator (Covaris M220, Covaris, Woburn, MA, USA) running the following program: duty factor of $20 \%$, peak power of 50 with 200 cycles/burst during $120 \mathrm{~s}$. Next, all samples were prepared for exome sequencing using a TruSeq DNA Sample Preparation Kit (Illumina) following the low-throughput, gel-free protocol according to the manufacture's instructions. DNA exonic sequences were enriched using the Truseq Exome Enrichment Kit (Illumina) according to the manufacturer's instructions. The enriched samples were hybridized and amplified on the three lanes of a pairedend flow cell using Illumina's cBot (Illumina). Next, all samples were exome sequenced using Illumina's HiSeq 1500 (Illumina) platform.

\section{Quality control and data analysis of whole-exome data}

Raw sequencing reads were analyzed using an in-house developed Perl-based workflow. First, FastQC Software (version 1.0) was used to assess quality of the raw data. Adapters and low-quality bases were trimmed using Cutadapt (version 1.2.1) and an in-house developed quality trimmer (Helsmoortel et al. 2014) respectively. Paired-end reads were then aligned to the human reference genome (hg19, NCBI Build 37) using a Burrows-Wheeler Aligner (BWA mem, version 0.7.4) (Li \& Durbin 2009). Picard (version 1.88) was used to mark and remove duplicates. Using the Genome Analysis ToolKit (GATK version 2.8.1) (McKenna et al. 2010), insertions and deletions (indels) were realigned and recalibrated.
Single-nucleotide variants (SNVs) and indels were detected using GATK, annotated with ANNOVAR (Wang et al. 2010), and filtered using VariantDB (Vandeweyer et al. 2014) according to different criteria (see Results section for details). Integrative Genomics Viewer (IGV, version 2.2.5) was used to visualize the reads (Thorvaldsdottir et al. 2012). Data were visualized using Circos Software (version 0.66) (Krzywinski et al. 2009) and made publicly available through European Nucleotide Archive (http://www.ebi.ac. uk/ena/data/view/PRJEB8223).

\section{Results}

\section{Determination of ploidy and chromosomal aberrations}

The genotyping results from the CytoSNP-12v2 Beadchip array were analyzed using the ASCAT algorithm, allowing correction for nondiploidy in samples from the two cell lines. ASCAT yielded an estimated average overall ploidy of 3 in BON-1 and 4 in QGP-1 (Fig. 1A and B). ASCAT analysis revealed multiple allelic duplications and deletions across the BON-1 and QGP-1 genome, resulting in frequent CNVs and regions with LOH (Fig. 1C and D). Long stretches of LOH were seen in chromosomes $1,3,4,6,9,11,17,18,22$, and $\mathrm{X}$ in BON-1, while in QGP-1, LOH of nearly entire chromosomes was observed in chromosomes 7, 8, 9, 13, 17, 21,22 , and $X$. These stretches of $\mathrm{LOH}$ were also seen in our next-generation sequencing (NGS) results (see below). In the NGS data, $\mathrm{LOH}$ was defined as an average allelic fraction (AF) above 0.66 in BON-1 and above 0.75 in QGP-1, corresponding to the respective ploidy of 3 and 4 (Fig. 2).

In order to confirm the ploidy calculations of ASCAT, we karyotyped both cell lines. GTG-banding results for BON-1 and QGP-1 were consistent with the results from the CytoSNP-12v2 Beadchip array. The BON-1 GTG-banding showed a near-triploid karyotype with a modal chromosome number of 63. Extensive chromosomal rearrangements and multiple derivative chromosomes were observed (Supplementary Figure S1, see section on supplementary data given at the end of this article). This was in accordance with results published previously (Lopez et al. 2010). GTGbanding of the QGP-1 cell line revealed a near-tetraploid karyotype with a modal chromosome numbers of 90-92, including multiple derivative chromosomes, thus confirming the ASCAT profile (Supplementary Figure S2, see section on supplementary data given at the end of this article).

\section{Detection and filtering of SNVs}

Whole-exome resequencing of BON-1 generated 91.5 million reads, while sequencing of QGP-1 generated

Published by Bioscientifica Ltd. 

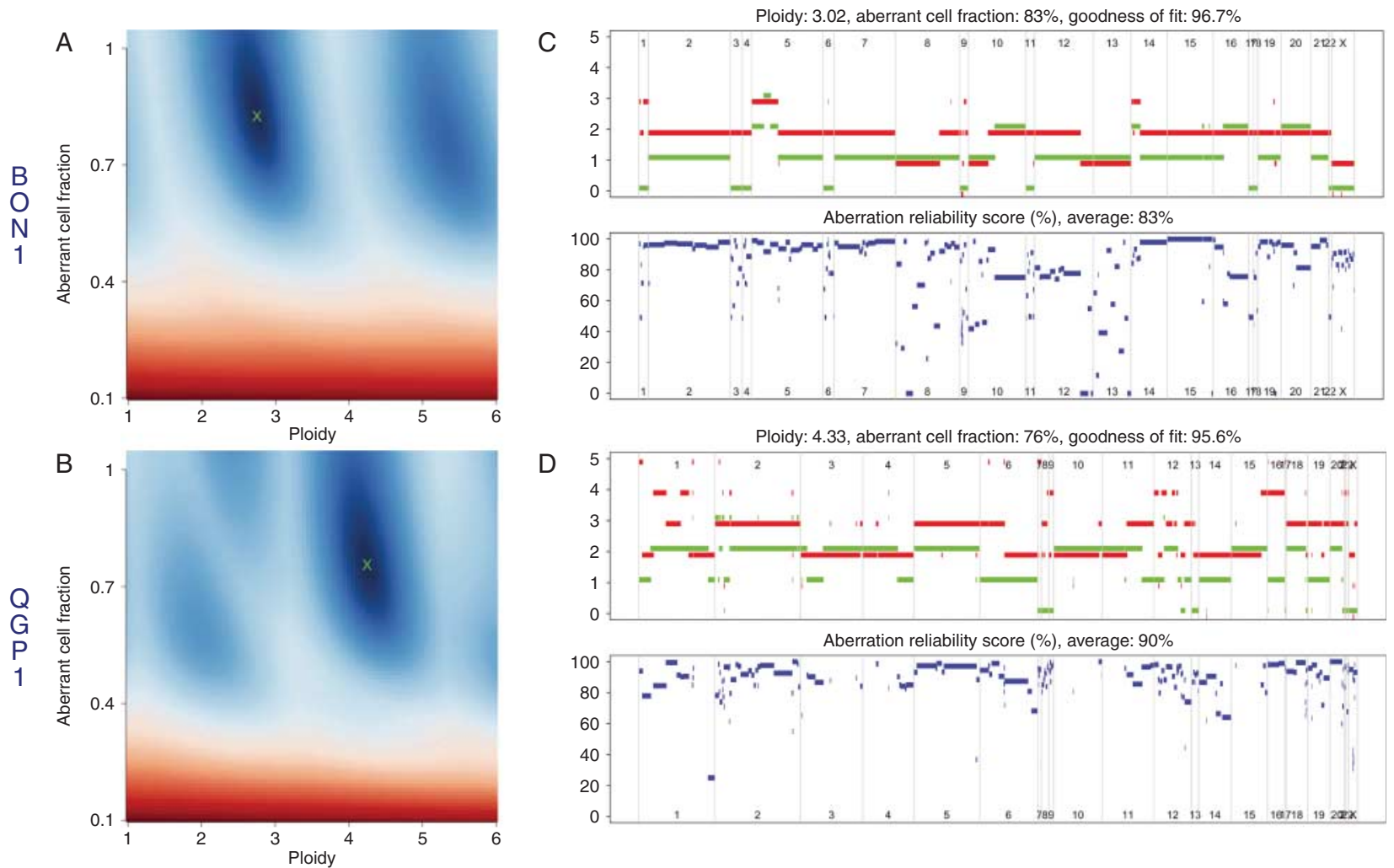

\section{Figure 1}

ASCAT profiles and their calculation. ASCAT first determines the ploidy of the tumor cells and the fraction of aberrant cells ( $A$ and $B$, sunrise plots). This procedure evaluates the goodness of fit for a grid of possible values for both parameters (dark, good solutions; pale, bad solutions). On the basis of this goodness of fit, the optimal solution is selected (crosses). The selected ploidy was 3 for BON-1 (A) and 4 for QGP-1 (B). Using the resulting tumor ploidy and aberrant cell fraction, an ASCAT profile was calculated ( $C$ and D, upper panels), containing the allele-specific copy number of all

107.6 million reads, Of these, $57.9 \%$ of the $\mathrm{BON}-1$ reads and $59.1 \%$ of the QGP-1 reads were mapped by BWA on targets enriched by the TruSeq Exome Enrichment Kit. The average base coverage for the target regions was $68 \times$ and $81 \times$ for BON-1 and QGP-1 respectively, with $92.9 \%$ of these nucleotides in BON-1 and 93.9\% in QGP-1 covered at least ten times (Fig. 2). After indel realignment, a GATK Unified Genotyper was used for variant detection with adapted settings for polyploidic samples. Based upon the SNP array and GTG-banding results, a ploidy of 3 for BON-1 and 4 for QGP-1 was selected as input for the GATK Unified Genotyper. GATK Unified Genotyper detected 65886 SNVs in BON-1 and 60979 SNVs in QGP-1 before filtering steps were applied (Fig. 3). Using VariantDB, we filtered these SNVs for a combination of criteria to reduce the likelihood of false-positive results (Fig. 3). All SNVs in snpEff noncoding regions were assayed loci. Copy number is shown on the $y$-axis vs the genomic location on the $x$-axis (light grey, allele with lowest copy number; dark grey, allele with highest copy number). For illustrative purposes only, both lines are slightly shifted (highest, down; lowest, up) such that they do not overlap. Only heterozygous SNPs are shown. Finally, for all aberrations that were found, an aberration reliability score was calculated ( $C$ and $D$, lower panels). A full colour version of this figure is available at http://dx.doi.org/ 10.1530/JME-14-0304.

removed from further analysis (Cingolani et al. 2012). To remove common SNPs, all variants present in the dbSNP137 (Sherry et al. 2001) and 1000 Genomes Project databases (1000 Genomes Project Consortium et al. 2010) with a minor allele frequency (MAF) higher than 0.05 were excluded. Filtering for RefSeq stoploss, stopgain, and nonsynonymous SNV (Pruitt et al. 2014) using VariantDB led to the identification of 1725 potentially proteinaltering SNVs in BON-1 and 1095 in QGP-1 (Table 1).

\section{Nucleotide substitution frequencies}

In both BON-1 and QGP-1, the most frequent nucleotide substitutions are $A \leftrightarrow G$ or $C \leftrightarrow T$ transitions (Fig. 4). In BON-1, transitions made up $66.8 \%$ of the filtered SNVs, resulting in a twofold overrepresentation of transitions compared with transversions. In QGP-1, transitions

Published by Bioscientifica Ltd. 

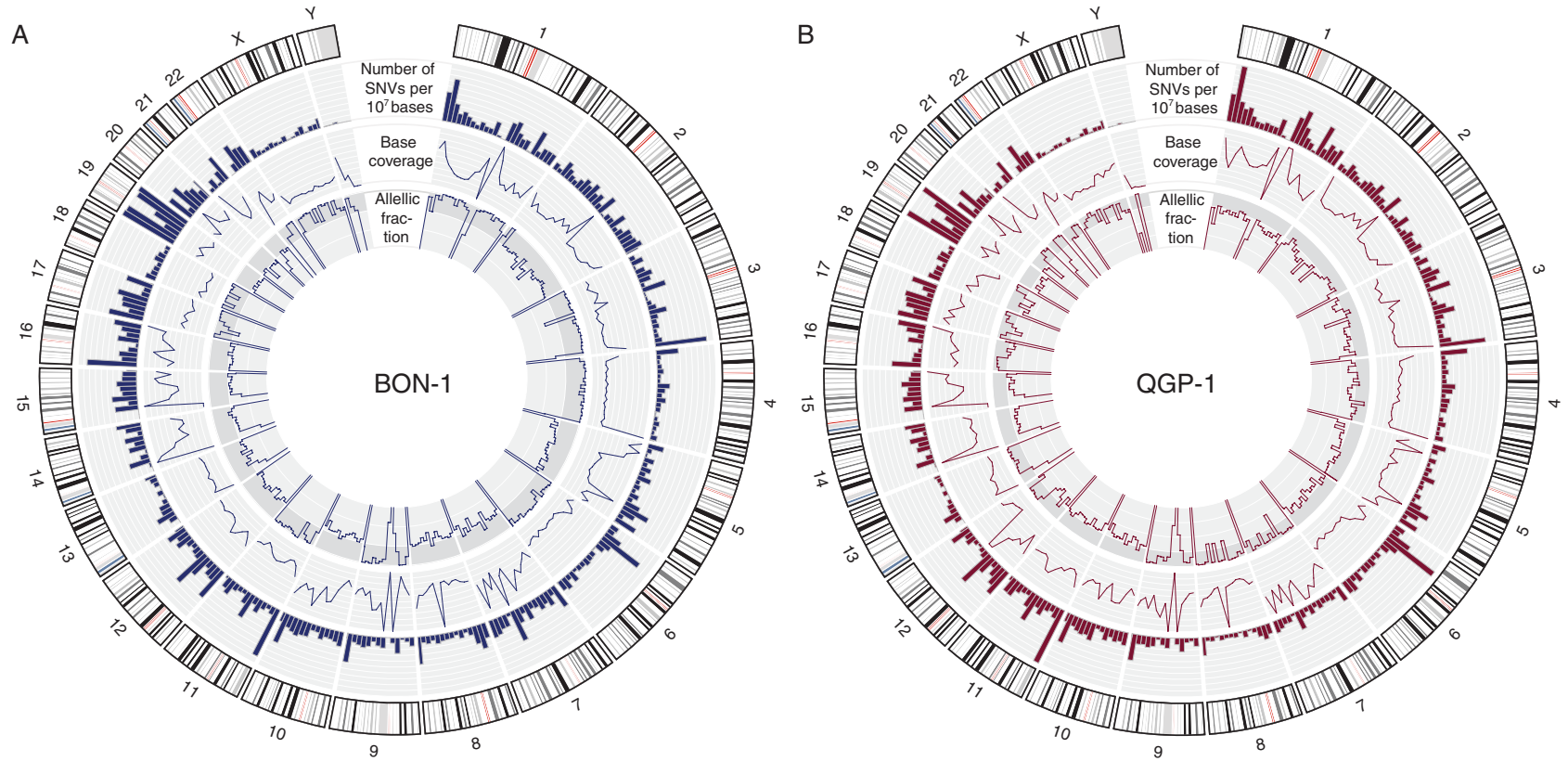

Figure 2

Circos plot showing number of predicted SNVs per $10^{7}$ bases (outer band, range $0-1000 \mathrm{SNVs} / 10^{7}$ bases), base coverage (middle band, range $0-200$ average base coverage over $10^{7}$ bases), and allelic fraction of the found SNVs (inner band, range $0-100 \%$ of reads show SNV, darker grey

accounted for $64.7 \%$ of the filtered SNVs. The ratio of transitions to transversions was 1:1.8 for the filtered SNVs in QGP-1. Overall, the ratio of transitions to transversions was similar in BON-1 and QGP-1 and higher than the average transitions to transversion rate of 1:1.7 that is usually seen in human germline samples (Lynch 2010). A mutation spectrum with a predominant $\mathrm{C}$ to $\mathrm{T} / \mathrm{G}$ to $\mathrm{A}$ transition pattern is seen in many adult cancers, including melanoma, breast, lung, colorectal, ovarian, and pancreatic adenocarcinomas (Greenman et al. 2007, Jones et al. 2008). However, in PNETs a more even distribution of transversions and transitions has been reported previously (Jiao et al. 2011).

\section{Detection of small indels}

On the indel-realigned and BWA-mapped reads, indels were identified using the GATK Unified Genotyper with the ploidy-settings adjusted to 3 for BON-1 and 4 for QGP-1. Quality filtering was executed with VariantDB using similar criteria to those for SNV filtering (Fig. 5). In BON-1, from the initial 2923 predicted insertions and 3194 predicted deletions, 20 insertions and 37 deletions were, after quality filtering, identified as RefSeq frameshift alterations, possibly leading to protein alterations. Of the initial 2749

background denotes homozygosity) across the genome in BON-1 (A) and QGP-1 (B). A full colour version of this figure is available at http://dx.doi. org/10.1530/JME-14-0304.

predicted insertions and 2903 predicted deletions in QGP-1, 21 RefSeq frameshift insertions and 35 RefSeq frameshift deletions remained after quality filtering.

\section{Prediction of effects on protein function}

To further identify SNVs with effects on protein function and a possible role in oncogenesis, in silico prediction of the effects on protein function and structure was made using four different software packages: PolyPhen2, MutationTaster, SIFT, and PROVEAN (Kumar et al. 2009, Adzhubei et al. 2010, Choi et al. 2012, Schwarz et al. 2014). In BON-1, 61.5\% of the filtered SNVs (1061 SNVs) had a predicted effect on protein function according to at least one of the four methods. In QGP-1, 67.2\% of all filtered SNVs (736 SNVs) were predicted to be deleterious with respect to protein function by at least one of the four packages used. However, only 137 SNVs $(7.9 \%$ of all filtered SNVs) were predicted to be deleterious by all four packages in BON-1, while 119 SNVs $(10.8 \%$ of all filtered SNVs) in QGP-1 were predicted by all packages to have a deleterious effect on protein function. MutationTaster was used for prediction of effects on proteins of frameshift indels in BON-1 and QGP-1. In BON-1, this identified 34 indels $(59.6 \%$ of identified indels) with a predicted

Published by Bioscientifica Ltd. 


\begin{tabular}{|c|c|c|}
\hline Filter & $\mathrm{BON}-1$ & QGP-1 \\
\hline Step 1: predicted alterations & $65886 \mathrm{SNVs}$ & 60979 SNVs \\
\hline Step 2: quality and coding DNA & 40728 SNVs & 37425 SNVs \\
\hline \multirow[t]{2}{*}{ Step 3: novel mutations } & 7819 SNVs & 3874 SNVs \\
\hline & $\nabla$ & $\nabla$ \\
\hline Step 4: protein-altering mutations & 1725 SNVs & 1095 SNVs \\
\hline
\end{tabular}

\section{Figure 3}

Number of single-nucleotide variants (SNVs) per filter step. Quality and coding DNA: total reading depth $\geq 10$, mapping quality $\geq 50$, Fisher-scaled strand bias $\leq 20$, allelic ratio $\geq 0.1$, Alternative allele-depth $\geq 2$, snpEff annotation $\neq$ noncoding; novel mutations: only SNVs with a MAF in NCBI dbSNP137 database $<0.05$ and in the 1000 genomes $2012<0.05$; proteinaltering mutations: RefSeq stopgain, stoploss, and nonsynonymous SNVs. A full colour version of this figure is available at http://dx.doi.org/10.1530/ JME-14-0304.

deleterious effect on protein function. In QGP-1, 23 indels ( $41.0 \%$ of identified indels) were predicted to be deleterious with respect to protein translation.

\section{Comparison of BON-1 and QGP-1 exomes}

When comparing the genomic positions of the identified SNVs and indels in BON-1 and QGP-1, 221 positions showed alterations in both QGP-1 and BON-1. Of these 221 genomic positions, QGP-1 and BON-1 showed the same nucleotide alterations in 216 positions (97.7\%) while at five positions a different nucleotide substitution, insertion, or deletion were seen. Of all the alterations found at these 221 genomic positions, 24 alterations were predicted by all four protein prediction programs to be damaging in QGP-1, while in BON-1, 25 alterations were predicted to be protein-damaging. We aimed at elucidating underlying PNET pathways and mechanisms by comparing BON-1 and QGP-1, looking at alterations at different genomic positions but within the same gene in BON-1 and QGP-1. First a list of genes containing SNVs or indels with a predicted damaging effect on protein function was created for BON-1 and QGP-1 separately. This resulted in a gene list containing 134 genes for BON-1 and 119 genes for QGP-1. When the two list were combined, 13 genes were identified that contained one or more mutations in both BON-1 and in QGP-1, including cancer-associated TP53 and CTBP2 (Takayama et al. 2014) (Supplementary Table 1, see section on supplementary data given at the end of this article).

\section{Genetic validation of SNVs}

Artifacts in next-generation whole-exome sequencing data could arise from different sources such as PCRamplification-based enrichment, sequencing technology proper or even sample mix-up. As we used a stringent filtering strategy, the risk was minimized, although not fully excluded. Therefore, using Sanger sequencing, we validated a subset of $22 \mathrm{SNV}$ and four indels from genes that came up as mutated in BON-1 and QGP-1 (Table 2). All SNVs and indels were confirmed by this technique. Although this represents only a small portion of all identified SNVs and indels, it illustrates the low level of sequencing artifacts in our filtered datasets.

\section{Discussion}

\section{Overview of genetic alterations}

The presented extensive genetic analysis of the neuroendocrine tumor cell lines BON-1 and QGP-1 is, to our knowledge, the first study to combine karyotyping, SNP array, and NGS whole-exome data. In a previous study, BON-1 was characterized using GTG-banding, comparative genomic hybridization (CGH), and fluorescence in situ hybridization (FISH) (Lopez et al. 2010). These lowresolution methods only allowed the study of large structural chromosomal alterations and copy number variations for a limited set of selected genes. SNVs cannot be identified by the methods applied in these studies. No data on the genetic constitution of QGP-1 are present in literature. Our study not only confirms previous findings regarding BON-1, but also adds new genotypic data using high-resolution methods. SNP array and NGS allow identification of small structural variations such as gene copy number variations and nucleotide changes such as SNVs and indels throughout the genome. For both BON-1 and QGP-1, this is the first time, to our knowledge, that the genome has been studied in this detail, leading to insights into the genetic origins of the two cell lines and their neuroendocrine tumor characteristics.

Table 1 RefSeq variant type of filtered SNVs in BON-1 and QGP-1

\begin{tabular}{|c|c|c|}
\hline $\begin{array}{l}\text { Variant-type } \\
\text { filtered SNVs }\end{array}$ & $\begin{array}{c}\text { BON-1 } \\
\text { (percentage } \\
\text { of total) }\end{array}$ & $\begin{array}{l}\text { QGP-1 } \\
\text { (percentage } \\
\text { of total) }\end{array}$ \\
\hline Stopgain SNVs & $33(1.9)$ & $26(2.4)$ \\
\hline Stoploss SNVs & $3(0.2)$ & $1(0.1)$ \\
\hline Nonsynonymous SNVs & 1689 (97.9) & $1068(97.5)$ \\
\hline All filtered SNVs & $1725(100)$ & $1095(100)$ \\
\hline
\end{tabular}

Published by Bioscientifica Ltd 


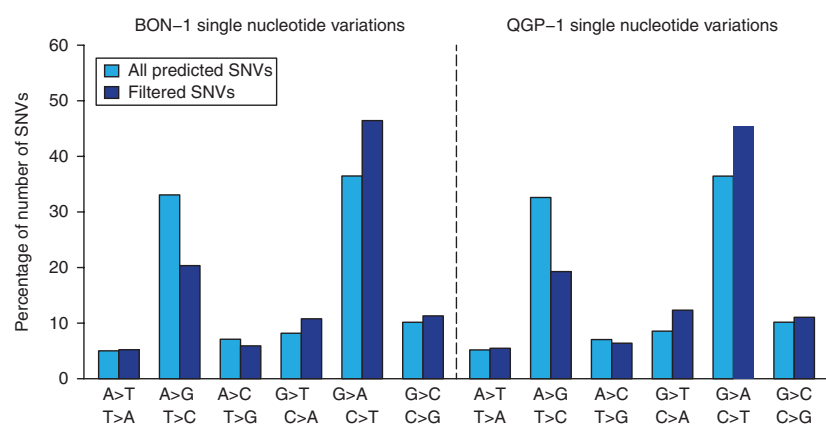

Figure 4

Mutation spectrum of single-nucleotide variants (SNVs) in BON-1 (left) and QGP-1 (right). Percentages of total number of predicted SNVs (light grey) and filtered SNVs (dark grey) in each of the six possible mutation classes. A full colour version of this figure is available at http://dx.doi.org/10.1530/ JME-14-0304.

An important characteristic is the nondiploid nature of the two cell lines. By using GTG-banding and a specialized ASCAT-algorithm for nondiploid SNP array data analysis, BON-1 could be accurately determined to be mainly triploid, which is in accordance with results reported previously (Lopez et al. 2010), and QGP to be mainly tetraploid. Adapting NGS variant identification algorithms to account for the nondiploidy made it possible to detect variants that were present in only one allele of the tri-allelic BON-1 or tetra-allelic QGP-1 cell line.

The first whole-exome data set of BON-1 and QGP-1 reveals a set of SNVs and indels that is not commonly present in the general population. This is indicated by the fact that $16.1 \%$ of all filtered SNVs and indels in BON-1 and 35.7\% in QGP-1 are not included in the publicly available dbSNP137 and 1000 Genomes Project databases. Germline DNA of the originating patients was not available, therefore it cannot be excluded that a minority of these variants are in fact rare germline SNPs that are not represented in these databases. However, given their predicted effects on protein function, we believe that the vast majority of filtered SNVs are not rare SNPs and, hence, might contribute to the tumorigenesis of PNETs.

\section{Extensive LOH in BON-1 and QGP-1}

In QGP-1 as well as BON-1, the SNP array and NGS results were indicative of an extensive $\mathrm{LOH}$ over multiple chromosomes. An increased frequency of LOH has been observed in 60 Caucasian human cancer cell lines derived from several organs and cell types including brain, CNS, colon, lung, white blood cells, melanocytes, ovary, prostate and kidney when compared with germline DNA of healthy subjects (Ruan et al. 2012). In addition, LOH in germline DNA is more frequent in patients with different tumor types in comparison with healthy controls (Assie et al. 2008). In a previous study, loss of CDKN2A and CDKN2B was identified in BON-1 through FISH (Lopez et al. 2010). The lack of reads in the NGS data at the respective $C D K N 2 A$ and $C D K N 2 B$ loci in BON-1 confirms these findings. $C D K N 2 A$ and $C D K N 2 B$ encode the tumor suppressors $\mathrm{p} 16^{\mathrm{INK} 4 \mathrm{~A}}$ and $\mathrm{p} 14^{\mathrm{ARF}}$, respectively, and inactivation could lead to unrestrained cell growth (Rocco \& Sidransky 2001). No CDKN2A and $C D K N 2 B$ alterations were found in QGP-1. In neuroendocrine tumors, results from a recent study have indicated the importance of LOH of the PHLDA3 gene in tumor progression and poor prognosis in addition to existing evidence of the importance of LOH of the MEN1 gene for tumorigenesis (Corbo et al. 2010, Ohki et al. 2014). Results from previous studies indicated that MEN1 expression was very low in BON-1 and that a reduction in growth was found when BON-1 was transfected with MEN1 (Stalberg et al. 2004). In this study, SNP array and NGS analysis of BON-1 yielded results indicative of $\mathrm{LOH}$ of MEN1. In addition, LOH of PHLDA3 was observed in BON-1. In QGP-1, LOH was observed for MEN1, but not for PHLDA3. This difference indicates that the two cell lines might have other mechanisms causing their malignant transformation.

\section{TP53 shows loss of function mutations in BON-1 and QGP-1}

p53 and associated proteins are important regulators of the cell cycle. In a previous study, exons 4-8 of

\begin{tabular}{|lcc|}
\hline \multicolumn{1}{l}{ Filter } & BON-1 & GGP-1 \\
\hline Step 1: predicted alterations & 6117 indels & \\
\hline & & \\
\hline Step 2: quality and coding DNA & 3969 indels & 3742 indels \\
\hline Step 3: novel mutations & & \\
\hline & 3963 indels & \\
\hline Step 4: protein-altering mutations & 57 indels & 56 indels \\
\hline
\end{tabular}

\section{Figure 5}

Number of insertions and deletions (indels) per filter step. Quality and coding DNA: total reading depth $\geq 10$, mapping quality $\geq 50$, Fisher-scaled strand bias $\leq 20$, allelic ratio $\geq 0.1$, snpEff annotation $\neq$ noncoding, Alternative allele-depth $\geq 2$; novel mutations: only SNVs and indels with a MAF in NCBI dbSNP137 database $>0.05$ and in the 1000 genomes 2012 $>0.05$; protein-altering mutations: RefSeq frameshift indels. A full colour version of this figure is available at http://dx.doi.org/10.1530/JME-14-0304.

Published by Bioscientifica Ltd. 
Table 2 SNVs confirmed by Sanger sequencing in BON-1 and QGP-1

\begin{tabular}{|c|c|c|c|c|}
\hline Target gene & Location & Reference allele & Alternative allele & Present in \\
\hline$A P C$ & chr5:112177788 & G & $A$ & QGP \\
\hline CYFIP2 & chr5:156721863 & $\mathrm{T}$ & $\mathrm{TC}$ & BON \\
\hline CYFIP2 & chr5:156816401 & $\mathrm{T}$ & A & QGP \\
\hline KANSL 1 & chr17:44144993 & $\mathrm{C}$ & G & BON \\
\hline KISS1 & chr1:204159611 & $\mathrm{CT}$ & $\mathrm{C}$ & QGP \\
\hline KISS1 & chr1:204159761 & G & C & BON \\
\hline KRAS & chr12:25398284 & $\mathrm{C}$ & A & QGP \\
\hline MAP4K2 & chr11:64559486 & G & $A$ & BON \\
\hline MAPK9 & chr5:179707528 & $\mathrm{T}$ & $\mathrm{C}$ & QGP \\
\hline MAPKBP1 & chr15:42111088 & C & $\mathrm{T}$ & BON \\
\hline MUC12 & chr7:100648713 & $\mathrm{C}$ & $\mathrm{T}$ & BON \\
\hline MUC16 & chr19:9018540 & $A$ & G & BON \\
\hline MUC16 & chr19:9057780 & C & $\mathrm{T}$ & QGP \\
\hline MUC17 & chr7:100677867 & $\mathrm{C}$ & $\mathrm{T}$ & QGP \\
\hline MUC17 & chr7:100681047 & C & $\mathrm{T}$ & QGP \\
\hline MUC17 & chr7:100684400 & G & A & BON \\
\hline MUC2 & chr11:1079672 & G & $A$ & BON \\
\hline MUC4 & chr3:195512107 & $\mathrm{T}$ & A & QGP \\
\hline PIK3C2A & chr11:17132074 & $A$ & G & QGP \\
\hline PTCH2 & chr1:45288988 & $\mathrm{C}$ & $\mathrm{T}$ & BON \\
\hline PTCH2 & chr1:45297968 & $A$ & G & QGP \\
\hline SMAD4 & chr18:48604787 & GACGA & G & BON \\
\hline TP53 & chr17:7574003 & G & $A$ & BON \\
\hline TP53 & chr17:7579393 & AG & A & QGP \\
\hline TSC2 & chr16:2134539 & $\mathrm{G}$ & $A$ & BON \\
\hline WNT1 & chr12:49375317 & C & $\mathrm{T}$ & BON \\
\hline
\end{tabular}

TP53 of BON-1 and QGP-1 were Sanger sequenced. This limited sequencing experiment failed to identify any mutations (Bartz et al. 1996). However, in our NGS sequencing data, we found a homozygous (AF 99\%) stopgain g.7574003A $>$ G mutation (ENSP00000269305 p.R342X) in exon 10 of TP53 in BON-1 and a homozygous (AF 100\%) g.7579394delG frameshift deletion (ENSP00000391127 p.P98Lfs*25) in exon 4 in QGP-1. Both mutations were validated through Sanger sequencing and predicted to be damaging by all four prediction algorithms. The predicted loss of function of p53 in the cell lines is supported by the previously reported lack of p53 mRNA and protein expression detectable by immunohistochemistry (Arany et al. 1994, Bartz et al. 1996). These findings imply that the two cell lines, at least partially, might rely on inactivation of p53 to escape cell death.

\section{Mutations in ATRX, RAS, and PI3K-AKT-mammalian target of rapamycin pathway genes}

Recent exome-sequencing efforts on tumor material from sporadic PNET patients have detected frequent mutations in ATRX, DAXX, and MEN1 (Jiao et al. 2011). ATRX and DAXX form a complex that plays a role in the incorporation of histone variant H3.3 at the telomeres, acting as epigenetic regulators (Lewis et al. 2010). MEN1 encodes menin, a nuclear protein important in chromatin remodeling and gene expression through histone acetylation and deacetylation (Kim et al. 2003). Conflicting results on the effects of $A T R X / D A X X$ alterations have been obtained. In one study, $A T R X / D A X X$ mutations correlated with a better survival in PNET patients with metastatic disease (Jiao et al. 2011). In other studies, with mainly early-stage-disease patients, loss of $A T R X / D A X X$ expression was associated with a worse prognosis (Marinoni et al. 2014, Yuan et al. 2014). In BON-1, the ATRX gene contains a homozygous (AF 100\%) g.77682471C > G missense mutation (ENSP00000362441 p.Q929E) and in QGP-1 a homozygous (AF 100\%) g.77682716A>G missense mutation (ENSP00000362441 p.F847S) was seen. The real effects of the two mutations on protein function are unknown, but all algorithms predicted them both to be benign. There were no mutations of the DAXX gene in BON-1 and QGP-1, confirming the mutually exclusive nature of $D A X X / A T R X$ mutations. Further functional studies are necessary to elucidate the role of the ATRX/DAXX pathway in BON-1 and QGP-1. Although in previous studies no MEN1 mutation was identified in BON-1 (Stalberg et al. 2004), our results revealed a missense homozygous (AF 100\%) g.64572018T > C MEN1

Published by Bioscientifica Ltd. 
SNP (ENSP00000377899 p.T546A) in both BON-1 and QGP-1. However, this SNP is benign according to the ClinVar database and thus probably does not play a role in the malign transformation of the BON-1 and QGP- 1 cells (Landrum et al. 2014).

In a previous study, a homozygous mutation in NRAS codon 61 was identified in BON-1 (Arany et al. 1994). In our BON-1 data, a homozygous (AF 100\%) g.114713908T > C mutation (ENSP00000358548 p.Q61R) was found in codon 61 of NRAS. In addition, a heterozygous g.25245350C $>$ A mutation (ENSP00000 308495 p.G12V) with an allelic ratio of 0.71 was found in the KRAS gene in QGP-1. Both the NRAS mutation in BON-1 and the KRAS mutation in QGP-1 were predicted to be damaging by all four prediction algorithms used. Results from a recent study have indicated that KRAS mutations are present in $10 \%$ of Chinese PNET patients and correlate with a poorer prognosis (Yuan et al. 2014). This might indicate a role of the RAS pathway in the oncogenesis of PNET.

The phosphoinositide-3-kinase/AKT/mammalian target of rapamycin (PI3K-AKT-mTOR) signaling pathway has been demonstrated to play a major role in NET by regulating cell growth, proliferation, survival, and protein synthesis (Kasajima et al. 2011). Hence, mTORinhibiting rapamycin and analogs (rapalogs) such as everolimus have a role in NET therapy. Results from a phase III trial were indicative of improved progressionfree survival with everolimus as monotherapy in progressive advanced PNET (Yao et al. 2011). However, in $15 \%$ of sporadic PNET mutations were found in PI3K-AKT-mTOR pathway-related genes such as PTEN, PI3KCA, and TSC2 (Jiao et al. 2011). No mutations were detected in the PI3K-AKT-mTOR pathway genes PTEN and $P I 3 K C A$ in BON-1 and QGP-1. However, three mis-sense mutations were detected in TSC2 in BON-1, including a g.2083797G $>$ A mutation (AF 0.63; ENSP00000344383 p.R1306H) predicted to be damaging by SIFT. This pattern of mutations could be indicative of a role of the TSC2 and, thus, the PI3K-AKT-mTOR pathway in the neoplastic proporties of the BON-1 cell line. In addition, comparing BON-1, a cell line with a TSC2 gene mutation, and QGP-1, a cell line without mutations in the PI3K-AKT-mTOR pathway, could elucidate the importance of these mutations in the efficacy of mTOR-directed drugs. Having a model for PNET with and without PI3K-AKT-mTOR pathway mutations could also be helpful for drug-development of targeted agents.

\section{Mucin as a possible new biomarker in PNET}

Mucins are high-molecular-weight glycoproteins with oligosaccharides attached to serine or threonine residues of the mucin core protein backbone by O-glycosidic linkages that are produced by various epithelial cells and play a role in cell-cell interactions (Yonezawa et al. 2008). MUC genes are highly variable in humans and often contain novel and unique SNVs in germline samples. For this reason, MUC mutations are sometimes removed from further analysis in studies of NGS data. However, mucin overexpression has frequently been seen in PNET tumor samples (Carrara et al. 2011, Carr et al. 2013). Hence, this study looked into the presence and relevance of MUC gene alterations in BON-1 and QGP-1. MUC4, MUC6, MUC16, and MUC17 genes showed possible protein-altering mutations in both cell lines. In addition, the MUC2, MUC5B, MUC12, and MUC13 genes show mutations with possible effects on proteins in BON-1. Further research is needed to fully understand the mechanisms that underlie the involvement of mucins in PNET.

In conclusion, the first, to our knowledge, highresolution genetic profile of the BON-1 and QGP-1 cell lines, based on array-based techniques and next generation whole-exome sequencing data, is presented in this article, greatly expanding on previous low-resolution genomic data for BON-1 and QGP-1 published previously. These frequently used PNET models show mutations in accordance with published findings regarding sporadic PNET in patients. However, further validation of BON-1 and QGP-1 as relevant genetic models for different subtypes of PNET in patients is needed. We believe that our data could contribute to a better understanding of the pathogenesis of PNET.

\section{Supplementary data}

This is linked to the online version of the paper at http://dx.doi.org/10.1530/ JME-14-0304.

Declaration of interest

The authors declare that there is no conflict of interest that could be perceived as prejudicing the impartiality of the research reported.

\section{Funding}

This work was supported by the Flemish Agency of Scientifc Research (FWO grant number G.0327.13N) and the European Neuroendocrine Tumor Society (ENETS)-Ipsen 2013 Translational Research Fellowship.

Published by Bioscientifica Ltd. 


\section{Acknowledgements}

The BON-1 cell line was a kind gift from J C Thompson, Department of Surgery, University of Texas Medical Branch, Galveston, TX, USA to L J Hofland, Section of Endocrinology, Department of Internal Medicine, Erasmus Medical Center, Rotterdam, The Netherlands for research purposes.

\section{References}

Adzhubei IA, Schmidt S, Peshkin L, Ramensky VE, Gerasimova A, Bork P, Kondrashov AS \& Sunyaev SR 2010 A method and server for predicting damaging missense mutations. Nature Methods 7 248-249. (doi:10.1038/nmeth0410-248)

Arany I, Rady P, Evers BM, Tyring SK \& Townsend CM Jr 1994 Analysis of multiple molecular changes in human endocrine tumours. Surgical Oncology 3 153-159. (doi:10.1016/0960-7404(94)90044-2)

Assie G, LaFramboise T, Platzer P \& Eng C 2008 Frequency of germline genomic homozygosity associated with cancer cases. Journal of the American Medical Association 299 1437-1445. (doi:10.1001/jama.299. 12.1437)

Baroni MG, Cavallo MG, Mark M, Monetini L, Stoehrer B \& Pozzilli P 1999 Beta-cell gene expression and functional characterisation of the human insulinoma cell line CM. Journal of Endocrinology 161 59-68. (doi:10.1677/joe.0.1610059)

Bartz C, Ziske C, Wiedenmann B \& Moelling K 1996 p53 tumour suppressor gene expression in pancreatic neuroendocrine tumour cells. Gut $\mathbf{3 8}$ 403-409. (doi:10.1136/gut.38.3.403)

Carr JC, Sherman SK, Wang D, Dahdaleh FS, Bellizzi AM, O'Dorisio MS, O'Dorisio TM \& Howe JR 2013 Overexpression of membrane proteins in primary and metastatic gastrointestinal neuroendocrine tumors. Annals of Surgical Oncology 20 (Suppl 3) S739-S746. (doi:10.1245/ s10434-013-3318-6)

Carrara S, Cangi MG, Arcidiacono PG, Perri F, Petrone MC, Mezzi G, Boemo C, Talarico A, Cin ED, Grassini G et al. 2011 Mucin expression pattern in pancreatic diseases: findings from EUS-guided fine-needle aspiration biopsies. American Journal of Gastroenterology 106 1359-1363. (doi:10.1038/ajg.2011.22)

Choi Y, Sims GE, Murphy S, Miller JR \& Chan AP 2012 Predicting the functional effect of amino acid substitutions and indels. PLOS ONE 7 e46688. (doi:10.1371/journal.pone.0046688)

Cingolani P, Platts A, Wang le L, Coon M, Nguyen T, Wang L, Land SJ, Lu X \& Ruden DM 2012 A program for annotating and predicting the effects of single nucleotide polymorphisms, SnpEff: SNPs in the genome of Drosophila melanogaster strain w1118; iso-2; iso-3. Fly 6 80-92. (doi:10.4161/fly.19695)

Corbo V, Dalai I, Scardoni M, Barbi S, Beghelli S, Bersani S, Albarello L, Doglioni C, Schott C, Capelli P et al. 2010 MEN1 in pancreatic endocrine tumors: analysis of gene and protein status in 169 sporadic neoplasms reveals alterations in the vast majority of cases. Endocrine-Related Cancer 17 771-783. (doi:10.1677/ERC-10-0028)

Doihara H, Nozawa K, Kojima R, Kawabata-Shoda E, Yokoyama T \& Ito H 2009 QGP-1 cells release 5-HT via TRPA1 activation; a model of human enterochromaffin cells. Molecular and Cellular Biochemistry 331 239-245. (doi:10.1007/s11010-009-0165-7)

1000 Genomes Project Consortium, Abecasis GR, Altshuler D, Auton A, Brooks LD, Durbin RM, Gibbs RA, Hurles ME \& McVean GA 2010 A map of human genome variation from population-scale sequencing. Nature 467 1061-1073. (doi:10.1038/nature09534)

Greenman C, Stephens P, Smith R, Dalgliesh GL, Hunter C, Bignell G, Davies H, Teague J, Butler A, Stevens C et al. 2007 Patterns of somatic mutation in human cancer genomes. Nature 446 153-158. (doi:10.1038/nature05610)

Helsmoortel C, Vandeweyer G, Ordoukhanian P, Van Nieuwerburgh F, Van der Aa N \& Kooy RF 2014 Challenges and opportunities in the investigation of unexplained intellectual disability using family-based whole-exome sequencing. Clinical Genetics (In Press). (doi:10.1111/cge. 12470)

Iguchi H, Hayashi I \& Kono A 1990 A somatostatin-secreting cell line established from a human pancreatic islet cell carcinoma (somatostatinoma): release experiment and immunohistochemical study. Cancer Research 50 3691-3693.

Jiao Y, Shi C, Edil BH, de Wilde RF, Klimstra DS, Maitra A, Schulick RD, Tang LH, Wolfgang CL, Choti MA et al. 2011 DAXX/ATRX, MEN1, and mTOR pathway genes are frequently altered in pancreatic neuroendocrine tumors. Science 331 1199-1203. (doi:10.1126/science.1200609)

Jones S, Zhang X, Parsons DW, Lin JC, Leary RJ, Angenendt P, Mankoo P, Carter H, Kamiyama H, Jimeno A et al. 2008 Core signaling pathways in human pancreatic cancers revealed by global genomic analyses. Science 321 1801-1806. (doi:10.1126/science.1164368)

Jonnakuty C \& Gragnoli C 2007 Karyotype of the human insulinoma CM cell line $-\beta$ cell model in vitro? Journal of Cellular Physiology $\mathbf{2 1 3}$ 661-662. (doi:10.1002/jcp.21135)

Kaku M, Nishiyama T, Yagawa K \& Abe M 1980 Establishment of a carcinoembryonic antigen-producing cell line from human pancreatic carcinoma. Gann 71 596-601.

Kasajima A, Pavel M, Darb-Esfahani S, Noske A, Stenzinger A, Sasano H, Dietel M, Denkert C, Rocken C, Wiedenmann B et al. 2011 mTOR expression and activity patterns in gastroenteropancreatic neuroendocrine tumours. Endocrine-Related Cancer 18 181-192. (doi:10.1677/ ERC-10-0126)

Kim H, Lee JE, Cho EJ, Liu JO \& Youn HD 2003 Menin, a tumor suppressor, represses JunD-mediated transcriptional activity by association with an mSin3A-histone deacetylase complex. Cancer Research 63 6135-6139.

Krzywinski M, Schein J, Birol I, Connors J, Gascoyne R, Horsman D, Jones SJ \& Marra MA 2009 Circos: an information aesthetic for comparative genomics. Genome Research 19 1639-1645. (doi:10.1101/gr.092759. 109)

Kulke MH, Siu LL, Tepper JE, Fisher G, Jaffe D, Haller DG, Ellis LM, Benedetti JK, Bergsland EK, Hobday TJ et al. 2011 Future directions in the treatment of neuroendocrine tumors: consensus report of the National Cancer Institute Neuroendocrine Tumor clinical trials planning meeting. Journal of Clinical Oncology 29 934-943. (doi:10.1200/JCO.2010.33.2056)

Kumar P, Henikoff S \& Ng PC 2009 Predicting the effects of coding non-synonymous variants on protein function using the SIFT algorithm. Nature Protocols 4 1073-1081. (doi:10.1038/nprot. 2009.86)

Landrum MJ, Lee JM, Riley GR, Jang W, Rubinstein WS, Church DM \& Maglott DR 2014 ClinVar: public archive of relationships among sequence variation and human phenotype. Nucleic Acids Research 42 D980-D985. (doi:10.1093/nar/gkt1113)

Leotlela PD, Jauch A, Holtgreve-Grez H \& Thakker RV 2003 Genetics of neuroendocrine and carcinoid tumours. Endocrine-Related Cancer 10 437-450. (doi:10.1677/erc.0.0100437)

Lewis PW, Elsaesser SJ, Noh KM, Stadler SC \& Allis CD 2010 Daxx is an H3.3-specific histone chaperone and cooperates with ATRX in replication-independent chromatin assembly at telomeres. PNAS 107 14075-14080. (doi:10.1073/pnas.1008850107)

Li H \& Durbin R 2009 Fast and accurate short read alignment with Burrows-Wheeler transform. Bioinformatics 25 1754-1760. (doi:10.1093/bioinformatics/btp324)

Lopez JR, Claessen SM, Macville MV, Albrechts JC, Skogseid B \& Speel EJ 2010 Spectral karyotypic and comparative genomic analysis of the endocrine pancreatic tumor cell line BON-1. Neuroendocrinology 91 131-141. (doi:10.1159/000254483)

Lynch M 2010 Rate, molecular spectrum, and consequences of human mutation. PNAS 107 961-968. (doi:10.1073/pnas.0912629107)

Marinoni I, Kurrer AS, Vassella E, Dettmer M, Rudolph T, Banz V, Hunger F, Pasquinelli S, Speel EJ \& Perren A 2014 Loss of DAXX and ATRX are associated with chromosome instability and reduced survival of 
patients with pancreatic neuroendocrine tumors. Gastroenterology 146 453-460 e455. (doi:10.1053/j.gastro.2013.10.020)

McKenna A, Hanna M, Banks E, Sivachenko A, Cibulskis K, Kernytsky A, Garimella K, Altshuler D, Gabriel S, Daly M et al. 2010 The Genome Analysis Toolkit: a MapReduce framework for analyzing nextgeneration DNA sequencing data. Genome Research 20 1297-1303. (doi:10.1101/gr.107524.110)

Ohki R, Saito K, Chen Y, Kawase T, Hiraoka N, Saigawa R, Minegishi M, Aita Y, Yanai G, Shimizu H et al. 2014 PHLDA3 is a novel tumor suppressor of pancreatic neuroendocrine tumors. PNAS 111 E2404-E2413. (doi:10.1073/pnas.1319962111)

Parekh D, Ishizuka J, Townsend CM Jr, Haber B, Beauchamp RD, Karp G, Kim SW, Rajaraman S, Greeley G Jr \& Thompson JC 1994 Characterization of a human pancreatic carcinoid in vitro: morphology, amine and peptide storage, and secretion. Pancreas 9 83-90. (doi:10.1097/ 00006676-199401000-00013)

Pruitt KD, Brown GR, Hiatt SM, Thibaud-Nissen F, Astashyn A, Ermolaeva O, Farrell CM, Hart J, Landrum MJ, McGarvey KM et al. 2014 RefSeq: an update on mammalian reference sequences. Nucleic Acids Research 42 D756-D763. (doi:10.1093/nar/gkt1114)

Rocco JW \& Sidransky D 2001 p16(MTS-1/CDKN2/INK4a) in cancer progression. Experimental Cell Research 264 42-55. (doi:10.1006/excr. 2000.5149)

Ruan X, Kocher JP, Pommier Y, Liu H \& Reinhold WC 2012 Mass homozygotes accumulation in the NCI-60 cancer cell lines as compared to HapMap Trios, and relation to fragile site location. PLOS ONE 7 e31628. (doi:10.1371/journal.pone.0031628)

Schimmack S, Svejda B, Lawrence B, Kidd M \& Modlin IM 2011 The diversity and commonalities of gastroenteropancreatic neuroendocrine tumors. Langenbeck's Archives of Surgery 396 273-298. (doi:10.1007/ s00423-011-0739-1)

Schwarz JM, Cooper DN, Schuelke M \& Seelow D 2014 MutationTaster2: mutation prediction for the deep-sequencing age. Nature Methods $\mathbf{1 1}$ 361-362. (doi:10.1038/nmeth.2890)

Seabright M 1971 A rapid banding technique for human chromosomes. Lancet 2 971-972. (doi:10.1016/S0140-6736(71)90287-X)

Shaffer LG, McGowan-Jordan J \& Schmid M 2009. ISCN2013: An International System for Human Cytogenetic Nomenclature (2009): Recommendations of the International Standing Committee on Human Cytogenetic Nomenclature, Basel: Karger AG.

Sherry ST, Ward MH, Kholodov M, Baker J, Phan L, Smigielski EM \& Sirotkin K 2001 dbSNP: the NCBI database of genetic variation. Nucleic Acids Research 29 308-311. (doi:10.1093/nar/29.1.308)

Silva SR, Bowen KA, Rychahou PG, Jackson LN, Weiss HL, Lee EY, Townsend CM Jr \& Evers BM 2011 VEGFR-2 expression in carcinoid cancer cells and its role in tumor growth and metastasis. International Journal of Cancer 128 1045-1056. (doi:10.1002/ijc.25441)
Stalberg P, Grimfjard P, Santesson M, Zhou Y, Lindberg D, Gobl A, Oberg K, Westin G, Rastad J, Wang S et al. 2004 Transfection of the multiple endocrine neoplasia type 1 gene to a human endocrine pancreatic tumor cell line inhibits cell growth and affects expression of JunD, $\delta$-like protein $1 /$ preadipocyte factor-1, proliferating cell nuclear antigen, and QM/Jif-1. Journal of Clinical Endocrinology and Metabolism 89 2326-2337. (doi:10.1210/jc.2003-031228)

Takayama K, Suzuki T, Fujimura T, Urano T, Takahashi S, Homma Y \& Inoue S 2014 CtBP2 modulates the androgen receptor to promote prostate cancer progression. Cancer Research 74 6542-6553. (doi:10.1158/0008-5472.CAN-14-1030)

Thorvaldsdottir H, Robinson JT \& Mesirov JP 2012 Integrative Genomics Viewer (IGV): high-performance genomics data visualization and exploration. Briefings in Bioinformatics 14 178-192. (doi:10.1093/bib/ bbs017)

Townsend CM Jr, Ishizuka J \& Thompson JC 1993 Studies of growth regulation in a neuroendocrine cell line. Acta Oncologica 32 125-130. (doi:10.3109/02841869309083900)

Vandeweyer G, Van Laer L, Loeys B, Van Den Bulcke T \& Kooy RF 2014 VariantDB: a flexible annotation and filtering portal for next generation sequencing data. Genome Medicine 6 74. (doi:10.1186/ s13073-014-0074-6)

Van Loo P, Nordgard SH, Lingjaerde OC, Russnes HG, Rye IH, Sun W, Weigman VJ, Marynen P, Zetterberg A, Naume B et al. 2010 Allelespecific copy number analysis of tumors. PNAS 107 16910-16915. (doi:10.1073/pnas.1009843107)

Wang K, Li M \& Hakonarson H 2010 ANNOVAR: functional annotation of genetic variants from high-throughput sequencing data. Nucleic Acids Research 38 e164. (doi:10.1093/nar/gkq603)

Yao JC, Hassan M, Phan A, Dagohoy C, Leary C, Mares JE, Abdalla EK, Fleming JB, Vauthey JN, Rashid A et al. 2008 One hundred years after "carcinoid": epidemiology of and prognostic factors for neuroendocrine tumors in 35,825 cases in the United States. Journal of Clinical Oncology 26 3063-3072. (doi:10.1200/JCO.2007.15.4377)

Yao JC, Shah MH, Ito T, Bohas CL, Wolin EM, Van Cutsem E, Hobday TJ, Okusaka T, Capdevila J, de Vries EG et al. 2011 Everolimus for advanced pancreatic neuroendocrine tumors. New England Journal of Medicine 364 514-523. (doi:10.1056/NEJMoa1009290)

Yonezawa S, Goto M, Yamada N, Higashi M \& Nomoto M 2008 Expression profiles of MUC1, MUC2, and MUC4 mucins in human neoplasms and their relationship with biological behavior. Proteomics 8 3329-3341. (doi:10.1002/pmic.200800040)

Yuan F, Shi M, Ji J, Shi H, Zhou C, Yu Y, Liu B, Zhu Z \& Zhang J 2014 KRAS and DAXX/ATRX gene mutations are correlated with the clinicopathological features, advanced diseases, and poor prognosis in Chinese patients with pancreatic neuroendocrine tumors. International Journal of Biological Sciences 10 957-965. (doi:10.7150/ijbs.9773)

Received in final form 16 January 2015

Accepted 22 January 2015

Accepted Preprint published online 22 January 2015 http://jme.endocrinology-journals.org DOI: 10.1530/JME-14-0304
() 2015 Society for Endocrinology Printed in Great Britain
Published by Bioscientifica Ltd. 